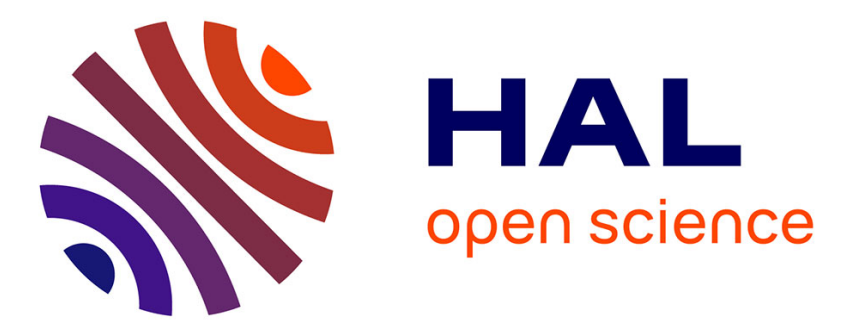

\title{
Influence of polarization mode competition on the synchronization of two unidirectionally coupled vertical-cavity surface-emitting lasers
}

Ignace Gatare, Marc Sciamanna, A. Locquet, Krassimir Panajotov

\section{- To cite this version:}

Ignace Gatare, Marc Sciamanna, A. Locquet, Krassimir Panajotov. Influence of polarization mode competition on the synchronization of two unidirectionally coupled vertical-cavity surface-emitting lasers. Optics Letters, 2007, 32 (12), pp.1629-1631. 10.1364/OL.32.001629 . hal-03079811

\section{HAL Id: hal-03079811 https://hal.science/hal-03079811}

Submitted on 17 Dec 2020

HAL is a multi-disciplinary open access archive for the deposit and dissemination of scientific research documents, whether they are published or not. The documents may come from teaching and research institutions in France or abroad, or from public or private research centers.
L'archive ouverte pluridisciplinaire HAL, est destinée au dépôt et à la diffusion de documents scientifiques de niveau recherche, publiés ou non, émanant des établissements d'enseignement et de recherche français ou étrangers, des laboratoires publics ou privés. 


\title{
Influence of polarization mode competition on the synchronization of two undirectionally coupled VCSELs
}

\author{
I. Gatare and M. Sciamanna \\ Supélec, LMOPS CNRS UMR-7132, 2 Rue Edouard Belin, F-57070, Metz, France
}

A. Locquet

Unité Mixte Internationale 2958 GeorgiaTech-CNRS, Georgia Tech Lorraine, 2 Rue Marconi, F-57070 Metz (France)

K. Panajotov

Department of Applied Physics and Photonics (TW-TONA), Vrije Universiteit Brussels,B-1050 Brussels, Belgium

Compiled November 24, 2006

We investigate theoretically the effect of polarization mode competition on the synchronization of two unidirectionally coupled vertical-cavity surface-emitting lasers (VCSELs). Chaos in the master laser is induced by delayed optical feedback and the slave laser is submitted to isotropic optical injection from the master VCSEL. We show that the synchronization quality can be enhanced when the chaotic regime in the master VCSEL involves both fundamental orthogonal linearly polarized modes. (c) 2006 Optical Society of America OCIS codes: $250.5270,260.5430$

The synchronization of chaotic systems has been a subject of both theoretical and experimental studies. ${ }^{1}$ In particular, the synchronization between two chaotic semiconductor lasers has attracted much attention owing to its interesting application in secure chaos-based optical communications. ${ }^{2}$ Among the diode lasers, verticalcavity surface-emitting lasers (VCSELs) are particularly interesting considering their intrinsic advantages such as a low threshold current, circular output beam with narrow divergence and the possibility to fabricate twodimensional laser arrays. Moreover, VCSELs may exhibit interesting polarization switching between two orthogonal linearly polarized modes when modifying their operating conditions such as injection current and temperature ${ }^{3}$ but also when they are subject to either optical injection ${ }^{4}$ or delayed optical feedback. ${ }^{5}$ In spite of this, studies on chaos synchronization in coupled VCSELs remain scarce. Recent theoretical works have focused on the synchronisation properties in polarization-dependent optical injection schemes ${ }^{6}$ or using multi-transverse but single LP mode VCSELs. ${ }^{7}$ Chaos synchronization in coupled VCSELs has also been very recently shown experimentally. ${ }^{8}$ The growing interest in implementing chaos communication using VCSELs motives further investigations in order to better understand the influence of polarization dynamics on the synchronization properties.

In this letter, we present a theoretical investigation of the effect of polarization mode competition on the synchronization characteristics of two unidirectionally coupled VCSELs. The master VCSEL only is rendered chaotic by optical feedback and its chaotic output is coupled to the slave VCSEL using an isotropic optical injection. Our results show that, depending on the injection conditions, the synchronization quality can be strongly enhanced when the master laser and therefore also the synchronized slave exhibit strong two LP mode dynamics.

The study of polarization dynamics and synchronization of the coupled VCSELs has been carried out using an extension of the standard Spin Flip Model (SFM) for single transverse mode VCSELs. ${ }^{9}$ The rate equations for the master and the slave lasers are:

$$
\begin{aligned}
\dot{E}_{x}^{m}= & \kappa(1+i \alpha)\left[\left(N^{m}-1\right) E_{x}^{m}+i n^{m} E_{y}^{m}\right] \\
& -\left(\gamma_{a}+i \gamma_{p}\right) E_{x}^{m}+f E_{x}^{m}(t-\tau) e^{i \omega_{m} \tau}+F_{x}^{m}(1) \\
\dot{E}_{y}^{m}= & \kappa(1+i \alpha)\left[\left(N^{m}-1\right) E_{y}^{m}-i n^{m} E_{x}^{m}\right] \\
& +\left(\gamma_{a}+i \gamma_{p}\right) E_{y}^{m}+f E_{y}^{m}(t-\tau) e^{i \omega_{m} \tau}+F_{y}^{m}(2) \\
\dot{E}_{x}^{s}= & \kappa(1+i \alpha)\left[\left(N^{s}-1\right) E_{x}^{s}+i n^{m} E_{y}^{s}\right] \\
& -\left(\gamma_{a}+i \gamma_{p}\right) E_{x}^{s}-i \Delta \omega E_{x}^{s}+\eta E_{x}^{m}(t)+F_{x}^{s},(3) \\
\dot{E}_{y}^{s}= & \kappa(1+i \alpha)\left[\left(N^{s}-1\right) E_{y}^{s}-i n^{m} E_{x}^{s}\right] \\
& +\left(\gamma_{a}+i \gamma_{p}\right) E_{y}^{s}-i \Delta \omega E_{y}^{s}+\eta E_{y}^{m}(t)+F_{y}^{s},(4) \\
\dot{N}^{m, s}= & -\gamma\left[N^{m, s}-\mu+N^{m, s}\left(\left|E_{x}^{m, s}\right|^{2}+\left|E_{y}^{m, s}\right|^{2}\right)\right] \\
& -i \gamma n^{m, s}\left(E_{y}^{m, s} E_{x}^{m, s^{*}}-E_{x}^{m, s} E_{y}^{m, s^{*}}\right), \\
\dot{n}^{m, s}= & -\gamma_{s} n_{s}^{m, s}-\gamma\left[n^{m, s}\left(\left|E_{x}^{m, s}\right|^{2}+\left|E_{y}^{m, s}\right|^{2}\right)\right] \\
& -i \gamma N^{m, s}\left(E_{y}^{m, s} E_{x}^{m, s^{*}}-E_{x}^{m, s} E_{y}^{m, s^{*}}\right) .
\end{aligned}
$$

The superscripts $m$ and $s$ are used for the master and slave VCSELs respectively. $E_{x}^{m, s}$ and $E_{y}^{m, s}$ are the slowly varying amplitudes of the $\mathrm{x}$ - and $\mathrm{y}$-LP field components. $N^{m, s}$ is the total population inversion between the conduction and the valence bands while $n^{m, s}$ accounts for the population difference between carrier densities with opposite spins. $\kappa$ is the photon decay rate, $\gamma$ is the decay rate of $N^{m, s}$, and $\gamma_{s}$ is a phenomenological parameter that accounts for microscopic processes leading to the homogenization of carriers with opposite spin values. $\alpha$ is the linewidth 
enhancement factor and $\mu$ is the normalized injection current ( $\mu=1$ at threshold). $\gamma_{a}$ and $\gamma_{p}$ model the linear cavity dichroism and phase anisotropy. $\Delta \omega=\omega^{m}-\omega^{s}$ is the frequency detuning. $f$ is the feedback rate, $\eta$ is injection rate. $\tau$ is the delay time in the external cavity. We consider a zero flight time between master and slave lasers. Spontaneous emission noise is taken into account by Langevin sources in the field equations: $F_{x}^{m, s}=$ $\sqrt{\beta_{s p} / 2}\left(\sqrt{N^{m, s}+n^{m, s}} \xi_{1}+\sqrt{N^{m, s}-n^{m, s}} \xi_{2}\right) \quad$ and $F_{y}^{m, s}=-i \sqrt{\beta_{s p} / 2}\left(\sqrt{N^{m, s}+n^{m, s}} \xi_{1}-\sqrt{N^{m, s}-n^{m, s}} \xi_{2}\right)$ with $\xi_{1}$ and $\xi_{2}$ two Gaussian white noises of zero mean and unitary variance. ${ }^{9}$ We keep the following parameters fixed: $\gamma=1 \mathrm{~ns}^{-1}, \gamma_{s}=50 \mathrm{~ns}^{-1}, \gamma_{a}=0.1 \mathrm{~ns}^{-1}$, $\kappa=300 \mathrm{~ns}^{-1} . \Delta \omega=0$ rad.ns ${ }^{-1}, \tau=3 \mathrm{~ns}, \mu=1.2$.

In our configuration, the two VCSELs can exhibit either an anticipating or isochronous (injection-locking) type of synchronization. ${ }^{10}$ Anticipating (perfect) synchronization is achieved when $I_{x, y}^{m}(t)=I_{x, y}^{s}(t-\tau)$, $N^{m}(t)=N^{s}(t-\tau)$ and $n^{m}(t)=n^{s}(t-\tau)$, with $I_{x, y}^{m, s}=\left|E_{x, y}^{m, s}\right|^{2}$. The necessary conditions for the existence of such a solution are that both lasers exhibit the same internal device parameters, bias currents, a zero detuning and also $f=\eta$. The isochronous synchronization solution is an injection-locked solution of the type: $I_{x, y}^{m}(t)=a I_{x, y}^{s}(t)$ with $a$ being a constant coefficient. To evaluate the synchronization quality in isochronous or anticipating synchronization conditions, we use the following correlation coefficients, respectively:

$$
\begin{aligned}
C_{1 x, y} & =\frac{\left[\left\langle I_{x, y}^{m}(t)-\left\langle I_{x, y}^{m}\right\rangle\right]\left[I_{x, y}^{s}(t)-\left\langle I_{x, y}^{s}\right\rangle\right]\right.}{\left\{\left\langle\left[I_{x, y}^{m}(t)-\left\langle I_{x, y}^{m}\right\rangle\right]^{2}\right\rangle\left\langle\left[I_{x, y}^{s}(t)-\left\langle I_{x, y}^{s}\right\rangle\right]^{2}\right\rangle\right\}^{\frac{1}{2}}}(7 .) \\
C_{2 x, y} & =\frac{\left[\left\langle I_{x, y}^{m}(t+\tau)-\left\langle I_{x, y}^{m}\right\rangle\right]\left[I_{x, y}^{s}(t)-\left\langle I_{x, y}^{s}\right\rangle\right]\right.}{\left\{\left\langle\left[I_{x, y}^{m}(t)-\left\langle I_{x, y}^{m}\right\rangle\right]^{2}\right\rangle\left\langle\left[I_{x, y}^{s}(t)-\left\langle I_{x, y}^{s}\right\rangle\right]^{2}\right\rangle\right\}^{\frac{1}{2}}}(8 .)
\end{aligned}
$$
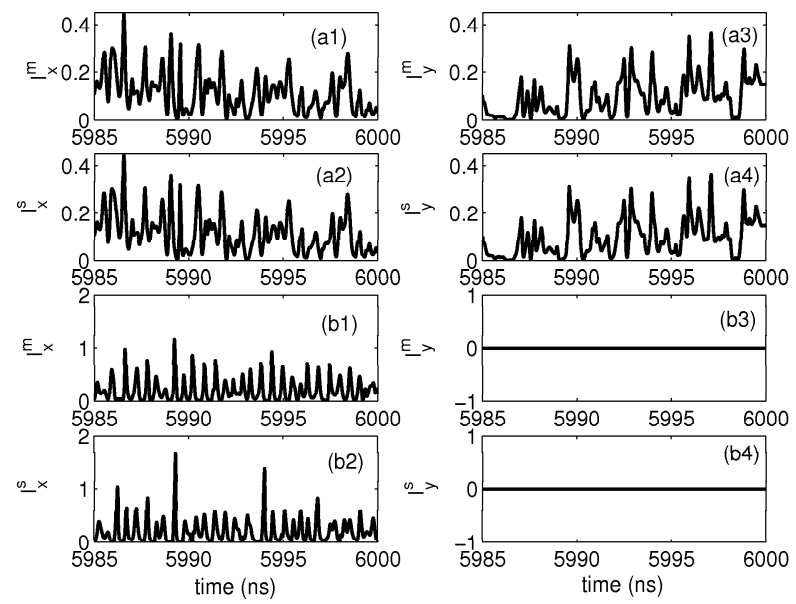

Fig. 1. Time-traces of master and slave laser LP mode intensities for $\eta=f=6 \mathrm{~ns}^{-1}$ : (a1-a4) chaos in both $\mathrm{x}$ and y-LP modes and (b1-b4) single-mode chaos.

To examine the effect of the polarization mode competition on the synchronization quality we consider first $\gamma_{p}=6 \mathrm{~ns}^{-1}$ and $\mu=1.2$ such that the free-running VC-
SELs operate in a parameter region where both $\mathrm{x}$ - and $\mathrm{y}-$ LP modes are stable. ${ }^{9}$ We first analyze mode competition without spontaneous emission noise (i.e. $\beta_{s p}=0$ ). With these parameters, we find that the dynamics induced by the delayed optical feedback in the master laser will also exhibit bistability. Depending on the system initial conditions, the master laser can exhibit either a chaotic dynamics involving both LP modes or a single mode chaotic dynamics, as shown in Figs. 1 (a1), (a3) and Figs. 1 (b1), (b3), respectively. We then analyze in each case the slave laser synchronization, as we progressively increase the injection rate. Without loss of generality we consider the injection and feedback conditions such that the necessary conditions for anticipative synchronization are met. Taking for example $\eta=f=6 \mathrm{~ns}^{-1}$ we observe first in Fig. 1 (a2), (a4) that the slave laser exhibits an almost perfect anticipative synchronization $\left(C_{2 x}=0.94\right)$ for each pair of corresponding LP modes. However, we find interestingly that for the same parameters but considering a single mode chaotic dynamics in the master laser leads to a significant decrease of the synchronization quality $\left(C_{2 x}=0.36\right)$; see Figs. 1 (b1), (b2).
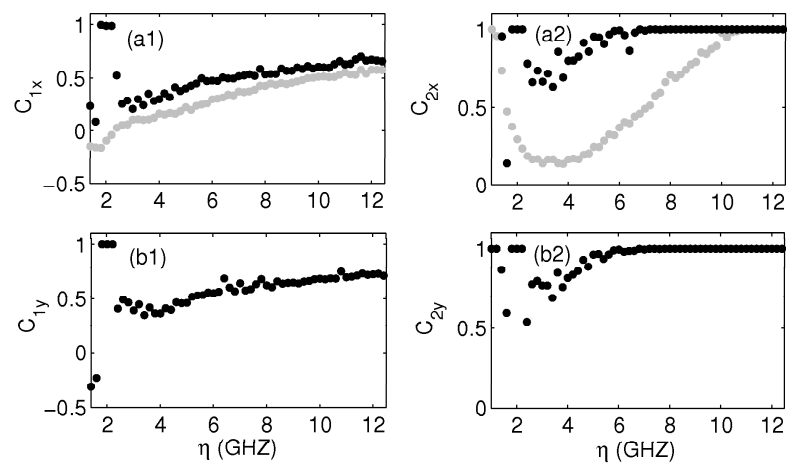

Fig. 2. Evolution of the correlation coefficients related to isochronous (a1 and b1) and anticipative (a2 and b2) synchronization as a function of $\eta$. The gray (black) color corresponds to the case of single-mode (two-mode) chaos.

In Fig. 2 we plot the evolution of both the isochronous and the anticipative correlation coefficients when the injection parameter $\eta$ is varied but still equal to the feedback rate $f$. We compare the case of two-LP mode chaotic dynamics (black) to that of single LP mode chaotic dynamics (gray). In both cases, the synchronization quality increases when $\eta$ increases and the anticipating synchronization quality is larger than the isochronous synchronization quality. In the case of two mode chaotic dynamics, the corresponding $\mathrm{x}-\mathrm{LP}$ modes synchronize as good as the corresponding y-LP modes; see the comparison between Figs. 2 (a1) and (b1) and between Figs. 2 (a2) and (b2). However, we find that both correlation coefficients $C_{1}$ and $C_{2}$ are relatively higher in the case of the two-mode chaotic dynamics; see Fig. 2(a1) and Fig. 2(a2) respectively. This effect of mode competition on the synchronization quality is more pronounced for the anticipative synchronization than for the isochronous 
synchronization. It is worth noting that, in the two-mode chaos regime and for $\eta<3 \mathrm{~ns}^{-1}$, the system may lock to a steady-state which explains the good isochronous synchronization.
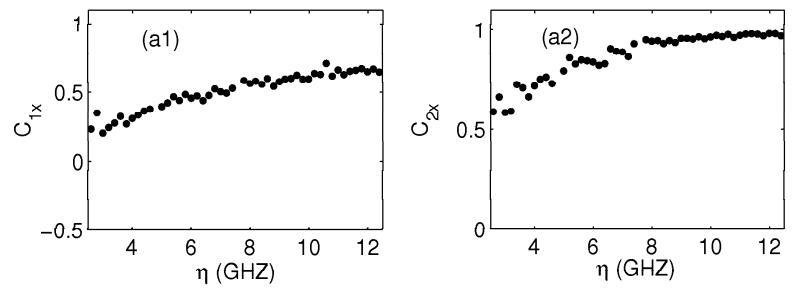

Fig. 3. Same as Fig. 2 but with $\beta_{s p}=10^{-6} \mathrm{~ns}^{-1}$.

If we now include the effect of spontaneous emission noise, we find that the master laser exhibits only a two mode chaotic dynamics, independently of the system initial condition. Figure 3 shows the evolution of the correlation coefficients $C_{1 x}$ and $C_{2 x}$, as a function of $\eta$ and for the same parameters than in Fig. 2 but $\beta_{s p}=10^{-6} \mathrm{~ns}^{-1}$. By comparing Fig. 3 to Fig. 2, we find that the synchronization quality is slightly degraded by the inclusion of the spontaneous emission noise, as also seen in other systems. ${ }^{?}$ However, both correlation coefficients are still higher than those obtained in the case of a deterministic single-mode chaotic dynamics [compare Figs. 3(a1) and 3(a2) to the gray curves in Figs. 2(a1) and 1(a2) respectively]. The enhancement of synchronization quality can therefore not be attributed to noise, which has typically the opposite effect, but rather to the excitation of two-mode dynamics.

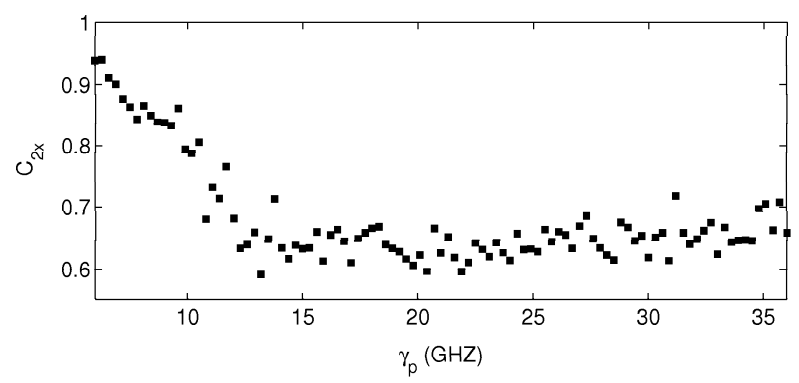

Fig. 4. Evolution of the anticipative synchronization quality when increasing the VCSEL birefringence. The other parameters remain the same than those in Fig. 3.

As a final test, we investigate how the anticipative synchronization quality evolves when the system is driven from its bistability region to a region where only a single mode solution exists even in the presence of spontaneous emission. In fact, we show that, by changing the VCSEL birefringence, a stable feedback-induced singlemode chaotic dynamics can be obtained. Fig. 4 shows the evolution of the system anticipative synchronization quality when the VCSEL birefringence $\gamma_{p}$ is changed but for a fixed injection parameter $\left(\eta=f=8 \mathrm{~ns}^{-1}\right)$. We start with $\gamma_{p}=6{\mathrm{rad} . n s^{-1}}^{-1}$ for which the system op- erates in a bistability region. As we have explained in the previous section (see the description of Fig. 3), the system exhibits a chaotic dynamics in both the orthogonal LP modes and a very good anticipative synchronization is achieved. If $\gamma_{p}$ is then increased, we observe an abrupt degradation of the synchronization around $\gamma_{p}=10 \mathrm{rad} . \mathrm{ns}^{-1}$. In fact at $\gamma_{p}=10{\mathrm{rad} . \mathrm{ns}^{-1}}^{-1}$, a dramatic change is found in the stability of the free-running VCSEL: for $\gamma_{p}>10{\mathrm{rad} . n \mathrm{~s}^{-1}}^{-1}$ only the $\mathrm{x}-\mathrm{LP}$ mode is stable. ${ }^{9}$ The system exhibits then a robust single mode dynamics even with noise and the decrease of synchronization quality corresponds to this transition from two mode to one mode dynamics. As $\gamma_{p}$ is still increased, the synchronization quality does not vary anymore.

In conclusion, we have shown that the synchronization quality between unidirectionally coupled VCSELs can be significantly enhanced when the feedback-induced chaos in the master laser involves both orthogonal LP fundamental transverse modes. This effect is particularly clear when tuning the VCSEL birefringence and even in the realistic presence of spontaneous emission noise. Our conclusions motivate dedicated experiments where the linear cavity anisotropies, and therefore the VCSEL polarization mode behavior, can be modified by strain and/or temperature effects. ${ }^{11}$ Our results are also thought to be important in the context of emerging VCSEL chaos based communication systems.

The authors acknowledge the support of Région Lorraine, COST 288, IAP "Photon" Network of the Belgian government, and by FWO-Flanders, GOA and OZR of the Vrije Universiteit Belgium.

\section{References}

1. L. M. Pecora, and T. L. Carroll, Phys. Rev. Lett. 64, 821 (1990).

2. C. R. Mirasso, P. Colet, and P. Garcia-Fernandez, IEEE Photon. Technol. Lett. 8, 2999 (1996).

3. K.D. Choquette, R.P. Scheneider, L.K. Lear and R.E. Liebenguth, IEEE J. Sel. Topics in Quantum Electron. 1, 661, (1995).

4. Z. G. Pan, S. Jiang, M. Dagenais, R. A. Morgan, K. Kojima, M. T. Asom, R. E. Leibenguth, G. D. Guth, and M. W. Focht, Appl. Phys. Lett. 63, 2999 (1993).

5. M. Sciamanna, K. Panajotov, H. Thienpont, I. Veretennicoff, P. Mégret, M. Blondel, Opt. Lett. 28, 1543 (2003).

6. R. Ju, P. Spencer, and K. A. Shore, IEEE J. Quantum Electron. 41 41, 1641 (2005).

7. M. S. Torre, C. Masoller, and K. A. Shore, J. Opt. Soc. Am. B 21, 1772 (2004).

8. Y. Hong, P. Spencer, and K. A. shore, Opt. Lett. 29, $1215(2004)$.

9. J. M. Regalado, F. Prati, M. San Miguel, and N. B. Abraham, IEEE J. Quantum Electron. 52, 765 (1997).

10. A. Locquet, F. Rogister, M. Sciamanna, P. Megret, and M. Blondel, Phys. Rev. E 64, 045203(R) (2001).

11. K. Panajotov, B. Nagler, G. Verschaffelt, A. Georgievski, H. Thienpont, J. Danckaert, and I. Veretennicoff, Appl. Phys. Lett. 77, 1590 (2000). 